\title{
Fivefold higher rate of pseudarthrosis with polyetheretherketone interbody device than with structural allograft used for 1-level anterior cervical discectomy and fusion
}

Presented at the 2018 AANS/CNS Joint Section on Disorders of the Spine and Peripheral Nerves

\author{
Katie L. Krause, MD, PhD, James T. Obayashi, BS, Kelly J. Bridges, MD, Ahmed M. Raslan, MD, \\ and Khoi D. Than, MD
}

Department of Neurological Surgery, Oregon Health \& Science University, Portland, Oregon

\begin{abstract}
OBJECTIVE Common interbody graft options for anterior cervical discectomy and fusion (ACDF) include structural allograft and polyetheretherketone (PEEK). PEEK has gained popularity due to its radiolucency and its elastic modulus, which is similar to that of bone. The authors sought to compare the rates of pseudarthrosis, a lack of solid bone growth across the disc space, and the need for revision surgery with the use of grafts made of allogenic bone versus PEEK.

METHODS The authors retrospectively reviewed 127 cases in which patients had undergone a 1-level ACDF followed by at least 1 year of radiographic follow-up. Data on age, sex, body mass index, tobacco use, pseudarthrosis, and the reoperation rate for pseudarthrosis were collected. These data were analyzed by performing a Pearson's chi-square test. RESULTS Of 127 patients, 56 had received PEEK implants and 71 had received allografts. Forty-six of the PEEK implants (82\%) were stand-alone devices. There were no significant differences between the 2 treatment groups with respect to patient age, sex, or body mass index. Twenty-nine (52\%) of 56 patients with PEEK implants demonstrated radiographic evidence of pseudarthrosis, compared to $7(10 \%)$ of 71 patients with structural allografts $(p<0.001, O R$ 9.82; $95 \% \mathrm{Cl} 3.836-25.139$ ). Seven patients with PEEK implants required reoperation for pseudarthrosis, compared to 1 patient with an allograft ( $p=0.01, \mathrm{OR} 10.00 ; 95 \% \mathrm{Cl} 1.192-83.884)$. There was no significant difference in tobacco use between the PEEK and allograft groups $(p=0.586)$.

CONCLUSIONS The results of this study demonstrate that the use of PEEK devices in 1-level ACDF is associated with a significantly higher rate of radiographically demonstrated pseudarthrosis and need for revision surgery compared with the use of allografts. Surgeons should be aware of this when deciding on interbody graft options, and reimbursement policies should reflect these discrepancies.
\end{abstract}

https://thejns.org/doi/abs/10.3171/2018.7.SPINE18531

KEYWORDS ACDF; anterior cervical discectomy and fusion; pseudarthrosis; PEEK; allograft

$\mathrm{A}$ NTERIOR cervical discectomy and fusion (ACDF) is one of the most common neurosurgical procedures performed for the treatment of cervical myelopathy and radiculopathy. ${ }^{1}$ Although immediate symptomatic relief is generally due to decompression of the affected neural structures, long-term success is dependent on the placement of an appropriate interbody graft within the disc space to maintain disc and foraminal height, restore cervical lordosis, and promote bone fusion..$^{11,13}$

As surgeons continue to refine this common procedure, options for graft material have increasingly multiplied. An autograft, often obtained from the patient's anterior iliac crest, is considered to be the gold standard due to its lack of histocompatibility difference from the removed disc,

ABBREVIATIONS ACDF = anterior cervical discectomy and fusion; $D B M=$ demineralized bone matrix; PEEK = polyetheretherketone; QALY = quality-adjusted life-year; rhBMP-2 = recombinant human bone morphogenetic protein-2.

SUBMITTED April 27, 2018. ACCEPTED July 19, 2018.

INCLUDE WHEN CITING Published online October 30, 2018; DOI: 10.3171/2018.7.SPINE18531. 
which could lead to graft rejection, and its ability to form a solid fusion construct. ${ }^{17,22}$ Harvesting bone for an autograft, however, comes with added morbidity, including donor site pain, stress fractures, and injury to the lateral femoral cutaneous nerve, as well as increased operative time, blood loss, and rate of surgical infection..$^{13,19,22}$ Allograft substitutes, including cortical, cancellous, and composite cadaver bone, have been employed to circumvent these complications, but they come with the theoretical risk of increased disease transmission, such as hepatitis and HIV, for which the estimated risks of disease spread are reported to be $0.01 \%$ and $0.03 \%$, respectively. ${ }^{6,14}$

More recently, synthetic interbody fusion devices have been developed, which are primarily made from carbon fiber, titanium, or polyetheretherketone (PEEK). ${ }^{28}$ The PEEK cage, in particular, has gained significant popularity due to its radiolucent properties and its elastic modulus, which is similar to that of bone., ${ }^{4,12}$ Furthermore, the use of PEEK cages results in increased billing per surgical level compared to allograft,,$^{23}$ which may further drive graft selection. Of note, for single-level cases, if a PEEK cage is used without a plate, the number of work relative value units is fewer than if a structural allograft is used with a plate (approximately 36 vs 49, depending on the payor). It seems conceivable that PEEK, a plastic material, would promote less bone fusion than a structural cadaveric bone allograft, even if the PEEK cage were packed with bone. Thus, we performed the largest retrospective cohort study to date to examine the incidence of radiographically demonstrated pseudarthrosis and subsequent reoperations in patients who underwent a 1-level ACDF with either a PEEK or structural allograft implant.

\section{Methods}

In this retrospective, single-center study, all consecutive 1-level ACDF procedures performed at the Oregon Health \& Science University between July 2011 and July 2016 were reviewed. Thirteen different attending surgeons ( 9 neurological surgeons and 4 orthopedic surgeons) performed the operative procedures. Any adult patient undergoing a 1-level ACDF for degenerative disease or trauma was included. Patients who did not have at least 1 year of follow-up with either a cervical x-ray study or CT scan were excluded. Implant selection, duration of follow-up, and the acquisition of follow-up imaging were dependent on the practice pattern of the individual surgeon. The study was approved by the local institutional review board, with a waiver of consent.

Electronic medical records were reviewed for demographic data, patient smoking status, type of graft material used, and evidence of pseudarthrosis. The presence of pseudarthrosis was defined as the lack of solid bone growth across the disc space at 1 or more years of radiographic follow-up. The primary investigators and an attending neuroradiologist independently reviewed all postoperative imaging studies. Records were further reviewed for any additional surgical intervention that was warranted beyond the index surgery. All records were also reviewed for the occurrence of postoperative infection.

Statistical analysis was undertaken using SPSS Statis- tics version 24 (IBM Corp.), and p values were considered significant at $<0.05$. Pearson correlation tests were used to determine whether there were statistically significant correlations between the rates of pseudarthrosis and of reoperations, and the graft materials (PEEK vs allograft materials). A Pearson correlation test was also used to determine if there was a statistically significant level of correlation between smoking history and graft material in patients in whom pseudarthrosis was confirmed. A Fisher exact test was used to determine the correlation between pseudarthrosis and the reoperation rate for PEEK grafts associated with a plate. A Student t-test was used to determine differences between the times of radiographic follow-up.

Mean results for the treatment groups are expressed as means \pm standard deviations.

\section{Results}

Four hundred eight patients underwent 1-level ACDF during the collection period; of these, 211 (51.7\%) received PEEK implants, $185(45.3 \%)$ received structural allograft implants, and $12(2.9 \%)$ received iliac crest autografts. Of the 408 patients, $127(31 \%)$ met the study's inclusion criteria: 56 (44\%) with PEEK implants and 71 (56\%) with structural allograft implants. The allograft implants included composite $(61 / 71)$, cortical $(8 / 71)$, or cancellous (2/71) materials. All PEEK cages were filled with nonstructural allograft in the form of demineralized bone matrix (DBM; 47/56) or a local autograft (9/56). The mean age of patients was $51 \pm 14.9$ years in the PEEK group and $53 \pm 13.0$ years in the allograft group. There was no significant difference in body mass index or smoking status between patients in the PEEK and allograft groups (Table 1). The overall $25 \%$ rate of smokers was slightly higher than the $17 \%$ rate in the overall US population. ${ }^{9}$ In both groups, the majority of procedures were performed for degenerative changes: 1 procedure was performed for trauma in the PEEK group (2\%) and 11 procedures were performed for trauma in the allograft group $(15.5 \%)(p=0.009)$. Excluding patients who underwent ACDF for trauma yielded similar pseudarthrosis rates: 27 (48.2\%) of 56 patients in the PEEK group and 5 (8\%) of 62 patients in the structural allograft group.

Patient imaging at the 1-year follow-up included x-ray studies in 110 patients $(86.6 \%)$ and CT scanning in 17 patients (13.4\%). In the PEEK group, $45(80.4 \%)$ of 56 pa-

TABLE 1. Patient demographics

\begin{tabular}{lccc}
\hline \multicolumn{1}{c}{ Factor } & $\begin{array}{c}\text { Structural } \\
\text { Allograft Group }\end{array}$ & $\begin{array}{c}\text { PEEK } \\
\text { Group }\end{array}$ & Total \\
\hline Patients & 71 & 56 & 127 \\
\hline Age in yrs (mean \pm SD) & $51 \pm 14.9$ & $53 \pm 13.0$ & $51.7 \pm 14.2$ \\
\hline Males & 34 & 21 & 55 \\
\hline Females & 37 & 35 & 72 \\
\hline Smokers & $17(24)$ & $15(27)$ & $32(25)$ \\
\hline BMl (mean \pm SD) & $28.4 \pm 0.6$ & $29.1 \pm 0.7$ & $28.7 \pm 0.6$ \\
\hline
\end{tabular}

Unless otherwise specified, values represent numbers of patients (\%, if given). There was no statistically significant difference between groups in any category. 
tients underwent x-ray studies compared to 65 (91.5\%) of 71 patients in the structural allograft group; the difference in these values was not statistically significant $(p=0.115)$. Average radiographic follow-up was longer in the PEEK group than in the structural allograft group: 21 versus 16 months, respectively $(\mathrm{p}=0.02)$. Of the 56 patients who received PEEK implants, 29 (51.8\%) had demonstrated radiographic evidence of pseudarthrosis at 1 or more years after follow-up, as seen on a cervical x-ray film or CT scan (Fig. 1). In contrast, only 7 (10\%) of the 71 patients with structural allograft implants had radiographic evidence of pseudarthrosis ( $\mathrm{p}<0.001$, OR 9.82; 95\% CI 3.8-25.1). Of patients with pseudarthrosis, 7 patients with PEEK implants $(24.1 \%)$ required a revision operation for pseudarthrosis, compared to only 1 patient with a structural al$\operatorname{lograft}(14.3 \%)(\mathrm{p}=0.01$, OR $10.00 ; 95 \%$ CI 1.192-83.884) (Table 2). Clinical indications for revision surgery for the 7 patients with PEEK implants included persistent radiculopathy (6/7), myelopathy (2/7), or chronic, debilitating neck pain (1/7). One of the 7 patients required revision surgery to correct completely fractured hardware with radiculopathy. The types of revision surgery included a redo $\mathrm{ACDF}$, a posterior instrumented fusion, and a combination of redo anterior fusion combined with posterior fusion.

The 1 patient who underwent revision ACDF surgery in the allograft group initially received a composite bone graft and displayed clinical indications of persistent radiculopathy. Interestingly, the graft for this patient was changed to a PEEK implant upon revision surgery. This was also the only patient in whom a postoperative wound infection developed after revision surgery; the infection was treated with operative washout and a course of antibiotics. There were no reports of postoperative transmission of hepatitis or HIV in either group.

The incidence of pseudarthrosis in patients who had received PEEK implants requiring plate and screw fixation was also examined. The majority of PEEK implants were stand-alone devices with no associated plate devices (46/56 implants, 82.1\%). Of the 10 patients who received PEEK implants with an associated plate, there was radiographic evidence of pseudarthrosis in 3 patients, 2 of whom required revision surgery. Compared to stand-alone PEEK implants, there was no significant correlation between a PEEK implant associated with a plate and the incidence of pseudarthrosis $(\mathrm{p}=0.171)$ or revision surgery $(p=0.596)$. In other words, PEEK implants led to higher pseudarthrosis rates than structural allografts regardless of whether the PEEK implants were stand-alone or supplemented with a plate and screws. However, the number of patients with a plated PEEK implant was very small $(\mathrm{n}=$ 10) and insufficient to draw strong conclusions.

Smoking status was further examined in patients with radiographic pseudarthrosis: 11 (37.9\%) of 29 patients with pseudarthrosis in the PEEK group smoked tobacco, whereas $4(57.1 \%)$ of 7 patients with pseudarthrosis in the allograft group smoked $(p=0.586)$ (Table 2). Of all patients with pseudarthrosis, only 1 patient in the PEEK group was on a long-term regimen of steroids for lupus.

\section{Discussion}

This retrospective study-the largest ever in which
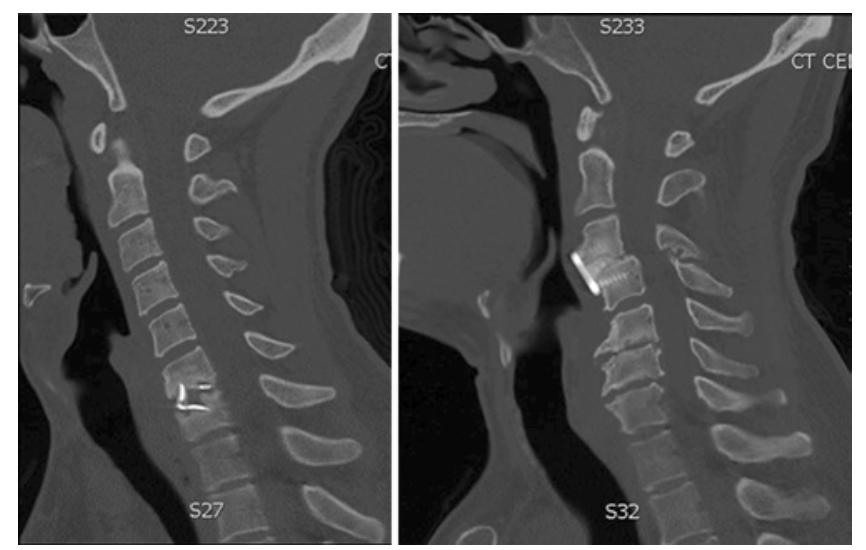

FIG. 1. Sagittal $x$-ray films obtained in a patient with a PEEK interbody graft and pseudarthrosis (left) and a patient with a structural allograft implant (right) healed 1 year after surgery.

PEEK implants have been compared with structural allografts for ACDF-demonstrates an alarmingly high rate of radiographic evidence of pseudarthrosis in patients who received PEEK grafts while undergoing a 1-level ACDF compared to those who received structural allografts. After at least 1 year of radiographic follow-up, there was a fivefold higher incidence of pseudarthrosis in patients with PEEK cages and almost a doubled rate of subsequent revision surgery.

Since their approval by the US Food and Drug Administration in 1998, ${ }^{12,21}$ PEEK implants have been a widely accepted choice as an interbody spacer. A recent study surveying 5334 surgeons from the Global AO Spine database found that PEEK cages make up $84 \%$ of cages selected for the graft component of an ACDF. ${ }^{28}$ PEEK implants have gained popularity because their elastic modulus is close to that of human bone, and in contrast to metallic cages, PEEK cages are composed of radiolucent material and produce less artifact on postoperative imaging. ${ }^{5}$ Furthermore, PEEK does not come with the risk of disease transmission that allograft spacers theoretically carry. However, the inherent bio-inertness of PEEK comes with the significant disadvantage of its being less likely to integrate with organic bone tissue..$^{21}$ In vitro studies have demonstrated that when mesenchymal cells are cultured on PEEK material, they do not express known markers of bone formation, including alkaline phosphatase or osteocalcin. ${ }^{15}$ Furthermore, mesenchymal cultures grown on PEEK have significantly higher levels of interleukin- $1 \beta$, which is associated

TABLE 2. Comparison of pseudarthrosis, need for revision surgery, and smoking status between the structural allograft and PEEK implant groups

\begin{tabular}{lccc}
\hline \multicolumn{1}{c}{ Factor } & $\begin{array}{c}\text { Structural } \\
\text { Allograft Group }\end{array}$ & $\begin{array}{c}\text { PEEK } \\
\text { Group }\end{array}$ & $\begin{array}{c}p \\
\text { Value }\end{array}$ \\
\hline Pseudarthrosis on imaging studies & $7(10)$ & $29(52)$ & $\leq 0.001$ \\
\hline Revision surgery & $1(14)$ & $7(24)$ & 0.01 \\
\hline Smokers w/ pseudarthrosis & $4(57)$ & $11(38)$ & 0.59 \\
\hline
\end{tabular}

Unless otherwise specified, values represent numbers of patients (\%). 
with the formation of fibrous tissue rather than bone tissue. Cells cultured on PEEK have also been demonstrated to have significantly higher levels of necrosis, DNA damage, and apoptosis. ${ }^{16}$ These in vitro studies are supported in an in vivo sheep model, which also demonstrated PEEK cages surrounded by fibrous connective tissue, preventing bone integration and potentially resulting in nonunion. ${ }^{24}$

In the clinical setting, there is little evidence for the superiority of PEEK over allograft, although studies describing well-controlled, direct comparisons between PEEK and allograft are limited. A recent meta-analysis found only 10 studies that directly compared PEEK to autograft, allograft, or other synthetic cages (titanium and carbon fiber). However, within those 10 studies there were no significant differences in fusion rates or clinical outcomes between PEEK and other graft materials. ${ }^{10}$ In only 2 of those 10 studies did researchers directly compare PEEK to allograft. Vaidya et al. ${ }^{25}$ performed a retrospective chart review of 46 consecutive cases of ACDF in which they compared patients treated with PEEK cages filled with recombinant human bone morphogenetic protein-2 (rhBMP-2) with patients treated with allograft interbody spacers and DBM at a single institution. Follow-up x-ray studies at 1.5-6 months postoperatively demonstrated that the PEEK cages filled with rhBMP-2 consistently exhibited $100 \%$ endplate resorption, which was said to have often been mistaken as infection by radiologists' interpretations. In contrast, there was no endplate resorption in any of the patients treated with allograft and DBM, with only "simple and progressive blurring" of the endplate junction, indicating ongoing fusion. However, at the 2-year followup, there was no significant difference in radiographic or clinical outcomes between the two groups, as measured by Cervical Oswestry Scale scores or visual analog scale scores. Subsequent cost analysis demonstrated that the cost of implants treated with PEEK and rhBMP-2 was more than 3 times the cost of those treated with allografts and DBM, which led the authors to ultimately abandon the use of PEEK and rhBMP-2 in lieu of the less expensive and equally effective allograft spacer. Another retrospective review ${ }^{20}$ compared PEEK and rhBMP-2 with allograft and rhBMP-2 for both ACDF and lumbar interbody fusion. In those patients who underwent an ACDF ( $\mathrm{n}=34)$, the PEEK and rhBMP-2 groups had slightly higher fusion rates than the allograft group (91\% vs 81\%, respectively), with 1 PEEK cage displaying cage migration. Similar to the findings of Vaidya et al., ${ }^{25}$ there was $100 \%$ endplate resorption with the use of rhBMP-2. There was a 50\% subsidence rate in all patients. ${ }^{20}$

This potential for subsidence is one main concern cited in the literature as a disadvantage of allografts, which can lead to loss of disc and foraminal height, increased angulation, and nonunion. ${ }^{2,3,18}$ However, in a recent retrospective study, researchers compared subsidence rates between PEEK and allograft cages and found that there was no significant difference between the PEEK (29\%) and allograft (28\%) groups. Furthermore, this study by Yson et al. demonstrated that even those patients who did have subsidence did not display any clinical difference from those who did not, as measured by the Neck Disability Index and the visual analog scale. ${ }^{29}$
Our findings have a wider implication on a systems level, as the number of ACDF procedures performed continues to increase, and reimbursement policies continue to evolve. Between 1992 and 2005, the rate of ACDFs grew by $206 \%$ in patients older than 65 years,${ }^{27}$ which is in line with the significant increase in general American healthcare spending, which rose to \$2.6 trillion in 2010 . As such, there has been increased scrutiny regarding the cost-effectiveness of all spinal procedures. ${ }^{26}$ In 1 study, a Markov decision model was used to determine the most effective graft (PEEK, allograft, or autograft) for a 1-level ACDF in terms of cost and quality of life. Cost was defined as the total sum of hospital, physician, and graft fees based on Current Procedural Terminology codes. The code designated for a PEEK interbody cage (22851) has a significantly higher reimbursement rate than that for a structural allograft (20931), ${ }^{23}$ with a work relative value unit of 6.7 versus 1.8 , respectively. As such, there was a significantly higher total cost for an ACDF with a PEEK cage (estimated total cost of $\$ 18,314$ ) than for the same procedure in which an allograft cage was used (estimated total cost of \$12,539). Virk et al. further examined the cost of quality-adjusted life-years (QALYs) gained by each graft type. PEEK was reported to be the most expensive, costing \$3220/QALY, compared to allograft at \$2358/ QALY and autograft at \$2413/QALY. ${ }^{26}$ This economic discrepancy is further widened by synthetic cage billing per level of placement, while allograft billing is once per surgery, regardless of the number of levels instrumented.

As Kersten et al. described in the review accompanying their meta-analysis, data regarding clinical outcomes of PEEK cages come mostly from noncomparative cohort studies and a few randomized control trials. ${ }^{10}$ Compared with data from previous studies, the advantage of the data we present here is that it offers a direct comparison of the incidence of radiographic pseudarthrosis between patients who received a PEEK cage and those who received a structural allograft in a 1-level ACDF. Furthermore, according to our review of previous studies, our study has the largest cohort of patients. However, our study does have limitations, which are inherent to its retrospective nature. A total of 13 different surgeons performed these procedures, making standardization of graft selection and the operative procedure difficult, although the similarity of the results across multiple surgeons does suggest generalizability of our findings. Differences between surgeons and changes in practice patterns were not evaluated. Confounders may stem from the lack of uniformity of physical graft placement, the type of structural allograft used (although the vast majority were composite), and the materials used to pack the PEEK cages (although the vast majority were packed with allograft and DBM). Many patients did not have 1 year of follow-up and thus were not included in the final analysis.

Another limitation of this study is that 2 different imaging modalities (x-ray and CT) were used to evaluate fusion. Ideally, all patients would have received goldstandard CT scanning, although the use of CT leads to increased costs as well as greater radiation exposure. One might even argue that complete bone bridging from endplate to endplate is not essential. As is the case with de- 
vices covered by plasma spray or sintered beads, PEEK does not undergo creeping bone substitution, as it just needs to be anchored at the ends to bone and will continue its load-bearing support irrespective of bone growth through the cage itself. As such, another imaging modality that could have been useful for assessing pseudarthrosis in this study, and which may be considered in future studies, is the flexion-extension x-ray study, which has been shown to provide a higher level of evidence for fusion. ${ }^{7}$

Also, as mentioned, the rate of cigarette smoking in the patient population of this study is slightly higher than the percentage of smokers in the overall US population, which may affect the generalizability of the results. In addition, the PEEK group in the present study also had a higher percentage of cigarette smokers than the structural allograft group. Although this finding was not statistically significant, it suggests that the two groups were not ideally matched. One should note, however, that the prevalence of smoking in patients with pseudarthrosis was higher in patients with structural allografts than in those with PEEK devices. This study is also lacking objective clinical data with validated outcome surveys, which will be a focus of future prospective studies. The ideal future study would be a multicenter study with a minimum of 2 years of followup and a better definition of the goal of the implants.

\section{Conclusions}

The results of this study suggest that the use of PEEK cages is associated with a significantly increased risk for bone nonunion and revision surgery compared to the use of structural allograft implants, at least at our institution. Thus, surgeons should consider these risks when deciding among the many graft choices available for an ACDF. Furthermore, reimbursement policies to reduce the cost discrepancy between PEEK and allograft should be advocated.

\section{Acknowledgments}

The authors thank Shirley McCartney, PhD, and Sharon Kinser for editorial assistance.

\section{References}

1. Bible JE, Kang JD: Anterior cervical discectomy and fusion: surgical indications and outcomes. Semin Spine Surg 28:80-83, 2016

2. Bishop RC, Moore KA, Hadley MN: Anterior cervical interbody fusion using autogeneic and allogeneic bone graft substrate: a prospective comparative analysis. J Neurosurg 85:206-210, 1996

3. Chau AMT, Mobbs RJ: Bone graft substitutes in anterior cervical discectomy and fusion. Eur Spine J 18:449-464, 2009

4. Chen F, He W, Mahaney K, Noeller J, Mhanna N, Viljoen S, et al: Alternative grafts in anterior cervical fusion. Clin Neurol Neurosurg 115:2049-2055, 2013

5. Cizek GR, Boyd LM: Imaging pitfalls of interbody spinal implants. Spine (Phila Pa 1976) 25:2633-2636, 2000

6. Delloye C, Cornu O, Druez V, Barbier O: Bone allografts: what they can offer and what they cannot. J Bone Joint Surg Br 89:574-579, 2007

7. Ghiselli G, Wharton N, Hipp JA, Wong DA, Jatana S: Prospective analysis of imaging prediction of pseudarthrosis after anterior cervical discectomy and fusion: computed to- mography versus flexion-extension motion analysis with intraoperative correlation. Spine (Phila Pa 1976) 36:463-468, 2011

8. Hee HT, Kundnani V: Rationale for use of polyetheretherketone polymer interbody cage device in cervical spine surgery. Spine J 10:66-69, 2010

9. Jamal A, Phillips E, Gentzke AS, Homa DM, Babb SD, King BA, et al: Current cigarette smoking among adults-United States, 2016. MMWR Morb Mortal Wkly Rep 67:53-59, 2018

10. Kersten RFMR, van Gaalen SM, de Gast A, Öner FC: Polyetheretherketone (PEEK) cages in cervical applications: a systematic review. Spine J 15:1446-1460, 2015

11. Kolstad F, Nygaard ØР, Andresen H, Leivseth G: Anterior cervical arthrodesis using a "stand alone" cylindrical titanium cage: prospective analysis of radiographic parameters. Spine (Phila Pa 1976) 35:1545-1550, 2010

12. Kurtz SM, Devine JN: PEEK biomaterials in trauma, orthopedic, and spinal implants. Biomaterials 28:4845-4869, 2007

13. Maharaj MM, Phan K, Mobbs RJ: Anterior cervical discectomy and fusion (ACDF) autograft versus graft substitutes: what do patients prefer?-A clinical study. J Spine Surg 2:105-110, 2016

14. Ng VY: Risk of disease transmission with bone allograft. Orthopedics 35:679-681, 2012

15. Olivares-Navarrete R, Gittens RA, Schneider JM, Hyzy SL, Haithcock DA, Ullrich PF, et al: Osteoblasts exhibit a more differentiated phenotype and increased bone morphogenetic protein production on titanium alloy substrates than on polyether-ether-ketone. Spine J 12:265-272, 2012

16. Olivares-Navarrete R, Hyzy SL, Slosar PJ, Schneider JM, Schwartz Z, Boyan BD: Implant materials generate different peri-implant inflammatory factors: poly-ether-ether-ketone promotes fibrosis and microtextured titanium promotes osteogenic factors. Spine (Phila Pa 1976) 40:399-404, 2015

17. Pollock R, Alcelik I, Bhatia C, Chuter G, Lingutla K, Budithi $\mathrm{C}$, et al: Donor site morbidity following iliac crest bone harvesting for cervical fusion: a comparison between minimally invasive and open techniques. Eur Spine J 17:845-852, 2008

18. Rhee JM, Patel N, Yoon ST, Franklin B: High graft resorption rates with dense cancellous allograft in anterior cervical discectomy and fusion. Spine (Phila Pa 1976) 32:29802984, 2007

19. Schnee CL, Freese A, Weil RJ, Marcotte PJ: Analysis of harvest morbidity and radiographic outcome using autograft for anterior cervical fusion. Spine (Phila Pa 1976) 22:22222227, 1997

20. Sethi A, Craig J, Bartol S, Chen W, Jacobson M, Coe C, et al: Radiographic and CT evaluation of recombinant human bone morphogenetic protein-2-assisted spinal interbody fusion. AJR Am J Roentgenol 197:W128-W133, 2011

21. Shimizu T, Fujibayashi S, Yamaguchi S, Otsuki B, Okuzu Y, Matsushita T, et al: In vivo experimental study of anterior cervical fusion using bioactive polyetheretherketone in a canine model. PLoS One 12:e0184495, 2017

22. Silber JS, Anderson DG, Daffner SD, Brislin BT, Leland JM, Hilibrand AS, et al: Donor site morbidity after anterior iliac crest bone harvest for single-level anterior cervical discectomy and fusion. Spine (Phila Pa 1976) 28:134-139, 2003

23. Singh K, Qureshi S: ISASS Policy Statement-cervical interbody. Int J Spine Surg 8:13, 2014

24. Toth JM, Wang M, Estes BT, Scifert JL, Seim HB III, Turner AS: Polyetheretherketone as a biomaterial for spinal applications. Biomaterials 27:324-334, 2006

25. Vaidya R, Carp J, Sethi A, Bartol S, Craig J, Les CM: Complications of anterior cervical discectomy and fusion using 
recombinant human bone morphogenetic protein-2. Eur Spine J 16:1257-1265, 2007

26. Virk SS, Elder JB, Sandhu HS, Khan SN: The cost effectiveness of polyetheretheketone (PEEK) cages for anterior cervical discectomy and fusion. J Spinal Disord Tech 28:E482E492, 2015

27. Wang MC, Kreuter W, Wolfla CE, Maiman DJ, Deyo RA: Trends and variations in cervical spine surgery in the United States: Medicare beneficiaries, 1992 to 2005. Spine (Phila Pa 1976) 34:955-963, 2009

28. Yoon ST, Konopka JA, Wang JC, Youssef JA, Meisel HJ, Brodke DS, et al: ACDF graft selection by surgeons: survey of AOSpine members. Global Spine J 7:410-416, 2017

29. Yson SC, Sembrano JN, Santos ERG: Comparison of allograft and polyetheretherketone (PEEK) cage subsidence rates in anterior cervical discectomy and fusion (ACDF). $\mathbf{J}$ Clin Neurosci 38:118-121, 2017

\section{Disclosures}

The authors have no personal, financial, or institutional interest in any of the drugs, materials, or devices described in this article. Dr. Raslan reports being a consultant to Abbott, and Dr. Than reports being a consultant to Bioventus.

\section{Author Contributions}

Conception and design: Than. Acquisition of data: Krause, Bridges. Analysis and interpretation of data: Than, Krause, Obayashi. Drafting the article: Than, Krause. Critically revising the article: Than, Krause, Bridges. Reviewed submitted version of manuscript: Than, Obayashi, Bridges, Raslan. Approved the final version of the manuscript on behalf of all authors: Than. Statistical analysis: Than, Krause, Obayashi, Raslan. Administrative/techni$\mathrm{cal} /$ material support: Than. Study supervision: Than.

\section{Supplemental Information}

\section{Previous Presentations}

Data shown in this report were presented by Dr. Krause at the Spine Summit 2018-34th Annual Meeting of the Section on Disorders of the Spine and Peripheral Nerves (Abstract no. 122, Top Abstracts Concurrent Session), March 14-17, 2018, Orlando, Florida; and in Abstracts of the 2018 AANS/CNS Joint Section on Disorders of the Spine and Peripheral Nerves Annual Meeting. Neurosurg Focus 44(3):A1-A109, 2018.

\section{Correspondence}

Khoi D. Than: Oregon Health \& Science University, Portland, OR.thank@ohsu.edu. 